\title{
CHIARITÉ
}

\section{Transparent Danionella translucida as a genetically tractable vertebrate brain model}

Lisanne Schulze, Jörg Henninger, Mykola Kadobianskyi, Thomas Chaigne, Ana Isabel Faustino, Nahid Hakiy, Shahad Albadri, Markus Schuelke, Leonard Maler, Filippo Del Bene, Benjamin Judkewitz

\section{Document type}

Postprint (accepted version)

This version is available at

https://doi.org/10.17169/refubium-29927

\section{Citation details}

Schulze L, Henninger J, Kadobianskyi M, Chaigne T, Faustino Al, Hakiy N, et al. Transparent Danionella translucida as a genetically tractable vertebrate brain model. Nature Methods. [Online] Springer Nature; 2018;15(11): 977-983. DOI: 10.1038/s41592-018-0144-6

\section{Terms of use}

All rights reserved. This document is intended solely for personal, non-commercial use. 


\title{
Transparent Danionella translucida as a genetically tractable vertebrate brain model
}

\author{
Lisanne Schulze ${ }^{*, 1}$, Jörg Henninger ${ }^{*, 1}$, Mykola Kadobianskyi ${ }^{1}$, Thomas Chaigne ${ }^{1}$, Ana Isabel \\ Faustino $^{1}$, Nahid Hakiy ${ }^{1}$, Shahad Albadri², Markus Schuelke ${ }^{1}$, Leonard Maler ${ }^{3}$, Filippo Del \\ Bene $^{2}$, Benjamin Judkewitz ${ }^{1,4}$ \\ 1. Charité - Universitätsmedizin Berlin, Einstein Center for Neurosciences, NeuroCure Cluster of Excellence, Berlin, Germany \\ 2. Institut Curie, Neuronal Circuit Development Group, Paris, France \\ 3. Brain and Mind Research Institute, Faculty of Medicine, University of Ottawa, Canada \\ 4. Humboldt University, Berlin, Germany \\ LS and JH contributed equally \\ Corresponding author: BJ (benjamin.judkewitz@charite.de)
}

\section{Abstract}

Understanding how distributed neuronal circuits integrate sensory information and generate behavior is a central goal of neuroscience. Yet, studying neuronal networks at single-cell resolution across the entire adult brain has been difficult in vertebrates due to their size and opacity. We address this challenge by introducing the fish Danionella translucida as a model organism to neuroscience. This teleost remains small and transparent even in adulthood, when neural circuits and behavior have matured. Despite its small brain, Danionella displays a rich set of complex behaviors, including courtship, shoaling, schooling and acoustic communication. To enable optical activity measurements and perturbations with genetically encoded tools, we established CRISPR/Cas9 genome editing and Tol2 transgenesis techniques. These features make Danionella trans/ucida a promising model organism for the study of adult vertebrate brain function at single-cell resolution. 


\section{Introduction}

Information processing in the brain is based on the complex interplay of large populations of neurons that are distributed across the nervous system. When neurons are integrated into circuits, functions emerge that cannot be predicted from the properties of individual cells. The study of neural networks is therefore essential for our understanding of higher brain functions such as perception, motor control, learning and social behavior.

Elucidating network function with cellular resolution is technically challenging. Technical advances in imaging resolution and speed, labelling and optogenetics ${ }^{1-7}$ have extended the use of optical techniques for measuring and perturbing neuronal population activity. Yet the size and opacity of vertebrate brains have prevented researchers from accessing the majority of neurons in the brain and forced them to focus on smaller regions.

An exception are zebrafish larvae which - with their genetic accessibility, small size and optical transparency - have become a successful vertebrate model system for studying distributed neuronal network function at whole-brain scale with single-cell resolution. This enabled network activity measurements and circuit analyses at a scale that has not been possible in other vertebrates ${ }^{8-16}$. However, larval networks are not yet mature and rewire over the course of development. Their early stage of brain development limits the study of complex behaviors that require an adult brain - including learning of complex tasks, courtship, communication and other social behaviors.

An ideal model organism would combine the advantages of invertebrates and zebrafish larvae - small brain size and optical access - with the behavioral and neural complexity of an adult vertebrate. Furthermore, such an organism should be easy to breed and amenable to genetic manipulation, including targeted gene knockout as well as insertion of transgenes. Here, we demonstrate that a freshwater Danionin fish species from Myanmar, Danionella translucida (DT), combines these properties. DT adults are among the smallest living vertebrates ${ }^{17}$, with a body length of about $12 \mathrm{~mm}$ (Figure $1 \mathrm{~A})$. . Bone anatomy studies ${ }^{18-21}$ suggested that the miniaturization in the genus Danionella is enabled by paedomorphic bone development.

Here, we show that DT adults have, to our knowledge, the smallest vertebrate brain. Despite their small size, we found a rich behavioral repertoire, including schooling, shoaling and other social behaviors. We report that DT males generate vocalizations with complex temporal 
structure. We genetically manipulated DT by adapting the Tol2 transposase system for gene insertion and by enabling CRISPR/Cas9 mediated inactivation of genes of interest by creating a sequencing library, which we aligned to the zebrafish genome. Using these tools, we created transgenic fish expressing the fluorescent calcium indicator GCaMP6f, and in proof-ofprinciple experiments we recorded spontaneous and auditory stimulus-evoked calcium signals.

\section{Results}

\section{Brain anatomy}

DT heads are transparent when observed under a stereomicroscope (Figure 1B): the hemispheres of the telencephalon and the optic tectum are visible in the intact head. The back of the eyes can be seen through the brain. This extent of optical clarity likely results from the absence of skull above the brain. Nissl-stained coronal sections through the head, which is covered only by skin, show that the skull surrounds the brain only laterally and ventrally (Figure 1C, Supplementary Video 1 , and ref. ${ }^{22}$ ). We estimated the total number of neurons in the DT brain to be 650000 (see Methods), which is over an order of magnitude lower than in adult zebrafish ${ }^{23}$. We next asked whether the reduction in neuron number is accompanied by a corresponding reduction of brain weight. However, attempting to weigh the brain or to determine brain volume from histological sections would lead to measurement errors resulting from a liquid film or from tissue shrinkage, respectively. We therefore resorted to $7 \mathrm{~T} \mathrm{MRI}$ of an intact DT specimen at $35 \mu \mathrm{m}$ isotropic resolution and determined the brain volume to be $0.6 \mathrm{~mm}^{3}$ (Supplementary Video 2, and ref. ${ }^{22}$ ). A comparison with the literature ${ }^{24-26}$ confirmed that DT has the smallest known adult vertebrate brain (Figure 1D).

\section{Behavior}

Although DT's small brain size and low number of neurons are desirable qualities for the optical study of neuronal networks, they raise the question of whether DT is capable of complex behaviors. We thus tested whether DT display behaviors known to require maturity in other fish species, such as courtship, shoaling, schooling and intra-species communication.

DT live in large schools and are communal breeders. We observed courtship episodes in silicon tubes in undisturbed community tanks during the first 5 hours of the light period. 
Typically, females linger inside the tubes when they are ready to spawn. Males approach females almost instantly after entering the tube and engage in apparent courtship behavior. After a short sequence of rapid back-and-forth movements and changes in orientation, the male and female come to rest with the male pushing against the posterior part of the female's body (time from male approach to egg release: median $=2 \mathrm{~s}$; range: 1 to $38 \mathrm{~s} ; \mathrm{n}=6$ ), and eggs are released (Supplementary Video 3). Females leave the tube shortly after spawning (time from spawning to female exit: median $=6 \mathrm{~s}$; range: 1 to $10 \mathrm{~s}$ ), while the male remains positioned on the egg clutch for several tens of seconds (time from spawning to male exit: median = $39 \mathrm{~s}$; range: 1 to $83 \mathrm{~s}$ ).

Shoaling and schooling are behaviours that play a key role in foraging, predator avoidance and mating strategies in fish ${ }^{27}$. Shoaling refers to groups of fish that remain together, while schooling describes shoals in which individuals show coordinated movement and aligned body orientation. We tested for shoaling and schooling in a group of 12 adult DT (aged 5 to 7 months) by monitoring their collective behavior in a shallow tank $\left(24 \times 24 \mathrm{~cm}^{2}\right.$, Figure $\left.2 \mathrm{~A}\right)$. To quantify the extent of shoaling we calculated the nearest-neighbor-distances for each pair and found that the average distance $(1.9 \pm 0.5 \mathrm{~cm}$, mean $\pm S D, n=15000$ frames, repeated twice with similar results, Figure $2 \mathrm{~B}$ ) was significantly smaller than the nearest-neighbor distance calculated under the assumption of complete spatial randomness $\left(3.6 \mathrm{~cm}, p=1.2 \cdot 10^{-3}\right.$ ClarkEvans Test) and average distance extracted from the shuffled dataset $(3.4 \pm 0.8 \mathrm{~cm}, \mathrm{p}<<$ $1 \cdot 10^{-5}$, Kolmogorov-Smirnov test). Additionally, fish coordinated their orientations as demonstrated by the non-uniform distribution of relative body orientations (Figure 2C). To test whether shoaling and schooling behaviors are visually mediated, we repeated the collective behavior recordings in darkness (under infrared illumination). Absence of visual cues abolished both shoaling and schooling (Supplementary Figure 1). In summary, DT adults showed visually mediated shoaling and schooling behavior.

A hallmark of social behavior is intra-species communication. While the majority of teleost fish communicate visually, hydrophone recordings confirmed that DT communicate via an additional modality: they generate vocalizations (Figure 2D-F, Supplementary Audio 1-2) consisting of short pulses $(<2 \mathrm{~ms})$ that are characterized by a sharp signal onset (Figure 2D) and amplitudes of around $140 \mathrm{~dB}$ (reference pressure: $1 \mu \mathrm{Pa}$, measured at distances $<13 \mathrm{~cm}$; max: $160 \mathrm{~dB}$ ) - acoustic signals audible without amplification even outside the tank. Pulses 
are generated predominantly at frequencies of about 60 and $120 \mathrm{~Hz}$, corresponding to peaks in the interpulse-interval histogram at about 8 and $17 \mathrm{~ms}$ (Figure 2E,G). They are structured in bursts ranging from about $30 \mathrm{~ms}$ up to minutes in duration (Figure 2E,F; distribution peak at $\sim 50 \mathrm{~ms}$, Supplementary Figure 2). Many bursts occur in clusters in which they are separated by intervals between about 60 to $300 \mathrm{~ms}$ and with total durations from tens of milliseconds up to minutes (distribution peak at $\sim 500 \mathrm{~ms}$, Figure 2E and Supplementary Figure 2B). In undisturbed DT community tanks, vocalizations occur frequently during the day, with emphasis on the first half of the day and coinciding with the hours of most frequent egg spawning, and only infrequently at night (Figure $2 \mathrm{H}$ ).

Only DT males generate vocalizations. We confirmed this by monitoring the vocalization behavior of small groups of defined sexes and found that female-only groups do not generate sounds (Figure 2I), in contrast to groups consisting of a single male and four females or of five males.

To investigate the potential function of vocalizations we next tested whether they are modulated by social context. If vocalizations were unrelated to social factors, their number would scale linearly with the number of fish in the tank. A nonlinear dependence would suggest social factors. To distinguish between these alternative hypotheses, we varied the number of males per tank and quantified the amount of vocalizations. The number of pulses generated by groups of males increases non-linearly with group size (Figure $2 \mathrm{~J}$ ), supporting a role of vocalizations in social behavior. Further quantification of behavior showed a notable cooccurrence of fighting behavior and vocalizations, suggesting a link between vocalizations and male-male aggression (Supplementary Figure 3).

\section{Genetic modification}

Genetic modification has become indispensable for biomedical research and is critical for widespread adoption of a new model organism. We therefore set out to develop transgenic techniques for DT. Our first goal was to express the genetically encoded calcium indicator GCaMP6f ${ }^{4}$ in neurons. We aimed to establish techniques already developed for other organisms with the hope of encouraging the adoption of DT by an existing community. A widely used tool for gene insertion in zebrafish and other teleosts is the Tol2-transposase system ${ }^{28}$. It requires the co-injection of Tol2 mRNA with a donor DNA plasmid into a single-cell stage 
embryo. To visualize activity in the adult DT brain, we used a construct expressing GCaMP6f under the zebrafish neurod1 promoter, which has previously been shown to lead to widespread expression in the adult zebrafish brain ${ }^{29}$. Injection of the DNA construct with Tol2 transposase into single-cell stage DT embryos (Figure 3A) led to mosaic expression in the nervous system (Figure 3B) in 40\% of injections (298 out of 735 fertilized embryos injected during a period of 6 months). Expression was prominent in the telencephalon and the cerebellum. We raised F0 larvae to adulthood (survival rate: $\sim 20 \%$ ), bred them in groups in home tanks, and confirmed germline transmission in the F1 generation (Figure 3C). We collected GCaMP6f-positive F1 from a permanent communal breeding tank with 30 adult F0 individuals exhibiting strong GCaMP6f expression. Over the course of 5 months, we collected 471 embryos in 40 clutches, of which 10 embryos in 6 clutches were GCaMP6f-positive (15\% of clutches and $2 \%$ of embryos). We did not perform single-pair matings as DT are communal breeders.

Having demonstrated transgene expression, we next sought to establish a gene knock-out strategy. We targeted pigmentation genes: while DT are mostly transparent, they have pigmented eyes and are sparsely covered by melanophores, which strongly absorb light (Figure 1). In zebrafish, a number of genes are known to control melanin pigmentation, including tyr (encoding tyrosinase), which can be inactivated using CRISPR/Cas9 ${ }^{30,31}$. To test whether we can use a similar strategy to remove the pigmentation in DT, we searched for a tyr gene homologue in the DT genome. We generated a short-read paired-end (100 bp) Illumina sequencing library and mapped reads to the zebrafish reference genome (Supplementary Figure 4). We found that DT reads aligned to at least one exon in over $85 \%$ of zebrafish genes, allowing for potential disruption of DT gene expression. To look for potential CRISPR/Cas9 targets in the DT tyr gene, we generated a consensus sequence of the first exon and selected two target sequences, tyr gRNA 1 and 2 (Figure 3D). To increase the probability of gene inactivation, we injected a mixture of these two gRNAs (Figure 3E). In $68 \%$ ( 49 out of 72 ) of embryos injected with tyr gRNA1+2 at the one-cell stage, we observed complete depigmentation (Figure 3F). We observed partial depigmentation when embryos were injected at the 2-4 cell stage $(n=6)$. We sequenced the targeted region in depigmented animals to confirm the knock-out in the tested gRNA combination. The gRNA target region was successfully cut with an efficiency of nearly $100 \%$ (Supplementary Figure 5). Furthermore, 
the injection of the gRNA pair led also to deletions between the two target sites of the expected size as determined by gel electrophoresis, which we also confirmed by sequencing (Supplementary Figure 5). To establish germline transmission of the genetic modifications, we assessed the depigmentation phenotype in F1 animals. Within 2 months, a breeding tank of 11 gRNA1+2 injected F0 adults laid 13 fertilized clutches with a total of 157 embryos. 12 clutches (92\%) containing 141 embryos were fully unpigmented (Figure 3G).

\section{Functional imaging}

To monitor neuronal activity throughout the brain in vivo, we performed two-photon microscopy in adult (3-6 months old) DT transiently expressing GCaMP6f under the neurod1 promoter. In order to keep brains stationary under the microscope, we devised an immobilisation strategy for DT. Zebrafish larvae are typically embedded inside a drop of agarose gel and continue to breathe through their skin. However, DT juveniles and adults require ventilation of the gills. We therefore used a modified strategy of partial agarose embedding to keep the gills free and employed low-level sedation to reduce motion artefacts without suppressing self-generated gill ventilation (Figure 4A-B).

With this strategy, we imaged individual GCaMP6f-expressing neurons down to a depth of 300 $\mu \mathrm{m}$ below the brain surface. As expected, Tol2 injected F0 individuals exhibited mosaic expression patterns (Figure 4C). In proof-of-principle calcium imaging experiments, we observed fluorescence intensity fluctuations in the telencephalon (Figure 4D,E) in 8 out of 18 animals. Of particular interest to us were hindbrain areas involved in auditory processing. In addition to mosaic expression, this area is difficult to image as it is covered by patches of melanophores above the hindbrain, since these individuals were wild type for tyr (see Figure $3 G$, left). In one F0 individual selected for expression in the medial hindbrain, 2 of 30 fluorescent cells exhibited auditory-evoked responses. We observed calcium activity of a neuron in the medial hindbrain reliably responding to auditory stimuli (bursts of 10 pulses at $125 \mathrm{~Hz}$, resembling bursts observed in natural vocalizations, Figure 4F,G).

\section{Data Resources}

In order to aid future research on DT, we make available three data resources alongside this publication: (1) a high-resolution $35 \mu \mathrm{m}$ isovoxel MRI scan of a DT specimen (83 MB), (2) Nissl-stained histological sections across the head, covering the entire brain (1.4 GB) and (3) 
the short-read genome sequencing alignment (3.6 GB, NCBI SRA \#SRP136594). Files and detailed descriptors can be found at http://danionella.org and ref. 22.

\section{Discussion}

In this work, we introduced a new model species with promising features for the study of vertebrate neural circuits.

DT generate vocalizations that are strongly modulated by social context, suggesting a function in communication. Intra-species communication signals are relevant, natural stimuli that in other fish species are employed in sex- and species-recognition, courtship behavior, mate assessment, resource defense, appeasement, alarm, and aggression ${ }^{33,34}$. Our finding that only males communicate is consistent with observations in Danionella dracula that only males have a putative sound generating organ, the "drumming apparatus" ${ }^{21}$. The basic unit of DT vocalizations is a single pulse. Pulses are arranged in a rich temporal structure spanning timescales from milliseconds to minutes, including bursts, burst sequences, and slow and fast pulse rates. We expect that this structure contains behaviorally relevant features and motifs that are extracted, detected and represented by dedicated circuits across auditory processing stages. The discrete nature of DT vocalizations makes them well suited for experimental manipulations. For example, behaviorally effective and ineffective abstractions of vocalizations will provide a powerful tool for investigating the neuronal computations underlying auditory feature detection. We note that auditory circuits are conserved across vertebrates ${ }^{35}$, and homologies between mammalian and fish brains have been suggested for the major auditory processing centers ${ }^{36-40}$.

Using conventional two-photon microscopy, we could discern individual GCaMP6f expressing neurons down to a depth of $300 \mu \mathrm{m}$ below the brain surface - reaching almost half of the brain volume. We expect that future work employing adaptive optics ${ }^{41-44}$ and longer wavelength light sources ${ }^{45,46}$ will be able to reach the entire brain at cellular resolution.

Having the smallest known adult vertebrate brain makes DT particularly attractive for investigations of vertebrate neuronal connectivity by volume electron microscopy (EM) ${ }^{47,48}$. At $0.6 \mathrm{~mm}^{3}$, the adult DT brain is only about $8 x$ larger than the Drosophila brain ${ }^{49}$ and contains about $6 \mathrm{x}$ as many neurons. A complete high-resolution EM volume of the Drosophila brain 
has been acquired within 16 months ${ }^{50}$. For DT, resolution requirements may be lower given that vertebrate synapses are monadic rather than polyadic and neuronal processes are larger ${ }^{16}$. DT thus brings the first adult vertebrate brain connectome within reach of existing imaging technology.

Not only biological factors influence the choice of model organism. Regardless of how suitable the biological features of a model species may be, one important consideration for its widespread adoption is the existence of established methods and a research community. When choosing an established model system, an investigator can benefit from available resources such as a body of literature, techniques and transgenic lines. As our work demonstrated, with DT we can take advantage of methods already developed by the zebrafish community. DT can be held in the same type of aquaria as zebrafish and fed the same food. Transgenic techniques are similar to zebrafish, and for gene insertion via Tol2, we could use identical plasmids for injection. CRISPR/Cas9 mediated gene editing cannot be performed with identical guide RNAs, but gRNA sequences can be designed based on the sequence library published with this paper ${ }^{22}$. Future work on de novo DT genome assembly and annotation will further expand the DT genetic toolkit.

In conclusion, an ultra-small brain, optical access and rich behavioral repertoire make DT particularly promising for investigations of neuronal network function in an adult vertebrate. 


\section{Author Contributions}

Conceptualization BJ, LS, JH; Methodology LS, JH, TC, MK, NH, AF, FDB, BJ; Software JH, MK, TC, BJ; Analysis JH, TC, MK, LS, SA, LM, BJ; Investigation LS, JH, AF, MK, SA, NH; Resources NH; Writing BJ, JH, with help from MK, LS, AF, TC; Supervision: MS co-supervised MK, LM co-supervised JH, FDB supervised SA; Overall supervision BJ

\section{Acknowledgments}

We thank Michael Brecht, Claire Wyart, Ralf Britz, Eva Naumann, Maximilian Hoffmann and Evgeny Bobrov for helpful discussions and for critically reading this manuscript. We are grateful to Pete Liptrot, Paul Dixon, Olaf Deters and other members of the Danionion aquarist community for advice on Danionella husbandry. Andrew Prendergast and Claire Wyart (ICM, Paris, France) advised us on the Tol2 strategy and kindly provided the NeuroD:GCaMP6f DNA construct prior to publication. Susan Luna (FishBase Project) kindly shared a digital copy of the FishBase brain data. We thank Susanne Mueller and the MRI core facility at Charite Berlin for providing their services and expertise. We acknowledge funding by the Einstein Foundation Berlin, the DFG (EXC 257 NeuroCure), and the Human Frontiers Science Program. BJ is a recipient of the Alfried Krupp Prize for Young University Teachers, awarded by the Alfried Krupp von Bohlen und Halbach-Stiftung.

\section{Competing interest statement}

The authors declare no competing interests. 


\section{References}

1. Betzig, E. et al. Imaging intracellular fluorescent proteins at nanometer resolution. Science 313, 1642-1645 (2006).

2. Bouchard, M. B. et al. Swept confocally-aligned planar excitation (SCAPE) microscopy for high speed volumetric imaging of behaving organisms. Nature Photonics 9, 113-119 (2015).

3. Tian, L. et al. Imaging neural activity in worms, flies and mice with improved GCaMP calcium indicators. Nat Meth 6, 875-881 (2009).

4. Chen, T.-W. et al. Ultrasensitive fluorescent proteins for imaging neuronal activity. Nature 499, 295-300 (2013).

5. Packer, A. M., Roska, B. \& Hausser, M. Targeting neurons and photons for optogenetics. Nat Neurosci 16, 805-815 (2013).

6. Ji, N., Freeman, J. \& Smith, S. L. Technologies for imaging neural activity in large volumes. Nat Neurosci 19, 1154-1164 (2016).

7. Chan, K. Y. et al. Engineered AAVs for efficient noninvasive gene delivery to the central and peripheral nervous systems. Nat Neurosci 20, 1172-1179 (2017).

8. Friedrich, R. W., Jacobson, G. A. \& Zhu, P. Circuit Neuroscience in Zebrafish Review. Curr Biol 20, R371-R381 (2010).

9. O'Malley, D. M., Kao, Y. H. \& Fetcho, J. R. Imaging the functional organization of zebrafish hindbrain segments during escape behaviors. Neuron 17, 1145-1155 (1996).

10. Orger, M. B., Kampff, A. R., Severi, K. E., Bollmann, J. H. \& Engert, F. Control of visually guided behavior by distinct populations of spinal projection neurons. Nat Neurosci 11, 327-333 (2008).

11. Ahrens, M. B. et al. Brain-wide neuronal dynamics during motor adaptation in zebrafish. Nature 485, 471-477 (2013).

12. Panier, T. et al. Fast functional imaging of multiple brain regions in intact zebrafish larvae using selective plane illumination microscopy. Front Neural Circuits 7, 65 (2013).

13. Portugues, R., Feierstein, C. E., Engert, F. \& Orger, M. B. Whole-Brain Activity Maps Reveal Stereotyped, Distributed Networks for Visuomotor Behavior. Neuron 81, 1328-1343 (2014).

14. Dunn, T. W. et al. Brain-wide mapping of neural activity controlling zebrafish exploratory locomotion. Elife 5, 471 (2016).

15. Naumann, E. A. et al. From Whole-Brain Data to Functional Circuit Models: The Zebrafish Optomotor Response. Cell 167, 947-+ (2016).

16. Hildebrand, D. G. C. et al. Whole-brain serial-section electron microscopy in larval zebrafish. Nature 545, 345-349 (2017).

17. Roberts, T. R. Danionella translucida, a new genus and species of cyprinid fish from Burma, one of the smallest living vertebrates. Environmental Biology of Fishes (1986).

18. Britz, R. Danionella mirifica, a new species of miniature fish from Upper Myanmar (Ostariophysi: Cyprinidae). Ichthyological Exploration of Freshwaters (2003).

19. Britz, R. Danionella priapus, a new species of miniature cyprinid fish from West Bengal, India (Teleostei: Cypriniformes: Cyprinidae). Zootaxa (2009).

20. Britz, R., Conway, K. W. \& Rüber, L. Spectacular morphological novelty in a miniature cyprinid fish, Danionella dracula n. sp. Proc. Biol. Sci. 276, 2179-2186 (2009).

21. Britz, R. \& Conway, K. W. Danionella dracula, an escape from the cypriniform Bauplanvia developmental truncation? J. Morphol. n/a-n/a (2015). doi:10.1002/jmor.20486

22. Schulze, L. et al., Dataset: Transparent Danionella translucida as a genetically tractable vertebrate brain model.(2018). doi: 10.12751/g-node.230a07

23. Hinsch, K. \& Zupanc, G. K. H. Generation and long-term persistence of new neurons in the adult zebrafish brain: a quantitative analysis. Neuroscience 146, 679-696 (2007).

24. Bauchot, M. L., Ridet, J. M. \& Diagne, M. Encephalization in Gobioidei (Teleostei). Japanese Journal of Ichthyology, 36(1), 63-74. (1989).

25. Froese, R. \& Pauly, D. FishBase. fishbase.org Available at: (Accessed: June 2017)

26. van Dongen, P. A. M. in The central nervous system of vertebrates 2099-2134 (Springer, Berlin, Heidelberg, 1998). doi:10.1007/978-3-642-18262-4_23

27. Pitcher, T. J. The Behaviour of Teleost Fishes. (Springer Science \& Business Media, 2012).

28. Kawakami, K. Tol2: a versatile gene transfer vector in vertebrates. Genome Biol. 8 Suppl 1, S7 (2007). 
29. Rupprecht, P., Prendergast, A., Wyart, C. \& Friedrich, R. W. Remote z-scanning with a macroscopic voice coil motor for fast 3D multiphoton laser scanning microscopy. Biomedical Optics Express 7, 1656-1671 (2016).

30. Jao, L.-E., Wente, S. R. \& Chen, W. Efficient multiplex biallelic zebrafish genome editing using a CRISPR nuclease system. Proc Natl Acad Sci USA 110, 13904-13909 (2013).

31. Di Donato, V. et al. 2C-Cas9: a versatile tool for clonal analysis of gene function. Genome Research 26, 681-692 (2016).

32. Hanken, J. \& Wake, D. B. Miniaturization of body size: organismal consequences and evolutionary significance. Annual Review of Ecology and ... 24, 501-519 (1993).

33. Bradbury, J. W. \& Vehrencamp, S. L. Principles of Animal Communication. (Sinauer Associates Incorporated, 2011).

34. Parmentier, E. \& Fine, M. L. in Vertebrate Sound Production and Acoustic Communication 1949 (Springer, 2016). doi:10.1007/978-3-319-27721-9_2

35. Grothe, B., Carr, C. E., Casseday, J. H., Fritzsch, B. \& Köppl, C. in Evolution of the Vertebrate Auditory System 22, 289-359 (Springer, New York, NY, 2004).

36. Mueller, T. What is the Thalamus in Zebrafish? Front. Neurosci. 6, 64 (2012).

37. McCormick, C. A. \& Wallace, A. C. Otolith end organ projections to auditory neurons in the descending octaval nucleus of the goldfish, Carassius auratus: a confocal analysis. Brain Behav Evol 80, 41-63 (2012).

38. Chagnaud, B. P., Engelmann, J., Fritzsch, B., Glover, J. C. \& Straka, H. Sensing External and Self-Motion with Hair Cells: A Comparison of the Lateral Line and Vestibular Systems from a Developmental and Evolutionary Perspective. Brain Behav Evol 90, 98-116 (2017).

39. Straka, H. Vestibular blueprint in early vertebrates. 1-9 (2013). doi:10.3389/fncir.2013.00182/abstract

40. Duncan, J. S. \& Fritzsch, B. Evolution of Sound and Balance Perception: Innovations that Aggregate Single Hair Cells into the Ear and Transform a Gravistatic Sensor into the Organ of Corti. Anat. Rec. 295, 1760-1774 (2012).

41. Ji, N., Milkie, D. E. \& Betzig, E. Adaptive optics via pupil segmentation for high-resolution imaging in biological tissues. Nat Meth 7, 141-147 (2010).

42. Wang, C. et al. Multiplexed aberration measurement for deep tissue imaging in vivo. Nat Meth 11, 1-7 (2014).

43. Wang, K. et al. Direct wavefront sensing for high-resolution in vivo imaging in scattering tissue. Nat Commun 6, 7276 (2015).

44. Papadopoulos, I. N., Jouhanneau, J.-S., Poulet, J. F. A. \& Judkewitz, B. Scattering compensation by focus scanning holographic aberration probing (F-SHARP). Nature Photonics 11, 116-123 (2017).

45. Horton, N. G. et al. In vivo three-photon microscopy of subcortical structures within an intact mouse brain. Nature Photonics 7, 205-209 (2013).

46. Ouzounov, D. G. et al. In vivo three-photon imaging of activity of GCaMP6-labeled neurons deep in intact mouse brain. Nat Meth 14, 388-390 (2017).

47. Denk, W. \& Horstmann, H. Serial Block-Face Scanning Electron Microscopy to Reconstruct Three-Dimensional Tissue Nanostructure. PLoS Biol 2, e329 (2004).

48. Denk, W., Briggman, K. L. \& Helmstaedter, M. Structural neurobiology: missing link to a mechanistic understanding of neural computation. Nat Rev Neurosci 4, 385 (2012).

49. Rein, K., Zöckler, M., Mader, M. T., Grübel, C. \& Heisenberg, M. The Drosophila Standard Brain. Current Biology 12, 227-231 (2002).

50. Zheng, Z. et al. A Complete Electron Microscopy Volume Of The Brain Of Adult Drosophila melanogaster. bioRxiv 140905 (2017). doi:10.1101/140905 


\section{Figure captions}

Figure 1 | Danionella translucida body size, transparency and miniature brain. (A) Images of a gravid female and male individual. (B) Image of the head. Black triangles indicate the position of brain sections shown in C. (C) Coronal sections of the DT brain. (D) Comparison of vertebrate brain weights versus body weights. Outlines are modified from ${ }^{26}$. Blue dots represent known adult fish, using data from ${ }^{24,25}$. Red dot: DT. Green dot: Zebrafish. Abbreviations. DM: dorsomedial telencephalon, DL: dorsolateral telencephalon, DC: central division of dorsal telencephalon, SP: subpallium, TeO: optic tectum, TS: torus semicircularis, CCe: corpus cerebelli, Hypo: hypothalamus, DFL: nucleus diffusus lateralis, CC: crista cerebellaris, nM: nucleus medialis, DON: dorsal octavolateral nucleus, RF: reticular formation.

Figure 2 | Behavioral repertoire of Danionella trans/ucida: (A) Motion traces of a DT school in a $24 \times 24 \mathrm{~cm}^{2}$ tank. (B) Nearest neighbor distances (blue). Bootstrapped distribution shown in red. The expected mean distance under the assumption of spatial randomness (see Methods) is shown as a gray line; $n=12$ fish. (C) Distribution of fish orientations relative to the mean orientation of all fish for each frame during schooling. Bootstrapped distribution shown in red, $n=12$ fish. D-F) Waveforms of vocalizations on different time scales. (D) DT males generate sequences of short, evenly spaced pulses. (E) Waveform of vocalizations of presumably a single male. The vocalizations are organized in sequences of bursts. (F) Vocalizations of various durations are frequent among groups of undisturbed animals. (G) Example distribution of intervals between the generated pulses. Example taken from a group of 5 animals. $(H)$ Diurnal distribution of vocalizations generated in community tanks. Shaded area depicts dark period, $n=7$ groups with 15-25 fish each. (I) Number of vocalization pulses generated over 24 hours by 5 animals versus sex composition of the studied group, $n=2$ groups for females and $n=4$ groups for mixed and male categories. $(J)$ Number of vocalization pulses generated over 24 hours by males versus the size of the studied group, $n=4$ for each category. Bars and error bars in I,J indicate mean and standard deviation. See also Supplementary Figure 1 and 2.

Figure 3 | Tol2 transgenesis and CRISPR/Cas9-mediated gene editing. (A) Schematic of Tol2-transposase mediated transgenesis. (B) Tol2 injected larvae showing transient GCaMP6f labeling. (C) F1 larva (16 dpf) carrying Tol2-mediated NeuroD:GCaMP6f transgene. Note that we used a bicistronic construct expressing RFP under the cryAA promoter in the lens as visible in the right eye (white star). The experiment was repeated more than 100 times with similar results. (D) Mapping of sequencing reads of DT to the Danio rerio reference genome at the first exon of the tyr gene (Coverage plot: grey - matching base, color - base mismatch). Target sequences for the CRISPR/Cas9 knockout are indicated by orange arrows. (E) Schematic of 
CRISPR/Cas9 and guide RNA injection into the single-cell stage embryo. (F) Melanin pigmentation of $3 \mathrm{dpf}$ wild-type DT larvae (left) and larvae injected with tyr gRNA1+2 target sequences and Cas9 at the 1-cell stage (middle, image was mirrored horizontally) and at the multiple (2-4) cell stage (right). (G) Pigmented wild type (left) and unpigmented tyr-mutant (right) DT individuals at 2 months post fertilization.

Figure 4 | Functional imaging of neural activity in GCaMP6f expressing Danionella translucida. (A) Schematic of the imaging setup (B) In vivo two-photon image of a DT specimen stained with the vital lipid dye Coumarin 6 to visualize brain regions (OB: olfactory bulb; Tel: Telencephalon; TeO: optic tectum; CB: cerebellum; HB: hindbrain) (C) Epifluorescence images of Tol2-injected DT adults expressing GCaMP6f (with labeled anterior-posterior axis and brain region outlines). (D) Two-photon image of GCaMP6f-positive neurons in the dorsolateral telencephalon. (E) Two-photon image of neurons in the medial hindbrain. (F) Spontaneous activity of GCaMP6f-positive neurons in the dorsolateral telencephalon. $(G)$ Acoustically evoked calcium-activity of neuron circled in $E$ (arrows indicate sound stimulus timing) recorded at $13.4 \mathrm{~Hz}$. Scale bars: $200 \mu \mathrm{m}$ in $\mathrm{B}, 500 \mu \mathrm{m}$ in $\mathrm{C}, 10 \mu \mathrm{m}$ in $D$ and $E$. 


\section{Methods}

Preparation of paraffin transverse sections

Male DT were sacrificed with an overdose of tricaine methanesulfonate (MS-222, $500 \mathrm{mg} / \mathrm{l}$ ) by prolonged immersion. The samples were then placed into ice-cold $4 \%$ paraformaldehyde for fixation, stored for at least two weeks at $4{ }^{\circ} \mathrm{C}$ for tissue permeation, and then prepared for sectioning according to the following procedure in collaboration with an external histology company (Morphisto $\mathrm{GmbH}$, Germany): fixative was washed out in phosphate buffered saline (PBS, 2.5h), 25\% EDTA solution for decalcification (72h), washing in PBS (2.5h), slow dehydration in ethanol in vacuum $(30 \%, 50 \%, 60 \%, 70 \%, 80 \%$ : $1.5 \mathrm{~h}$ each; $90 \%$ : $3.5 \mathrm{~h}, 96 \%$ : $5 \mathrm{~h})$, isopropanol (4h), xylol (6h), paraffin/xylol solution (1:1, 5h), paraffin (14h). Transverse 8 $\mu \mathrm{m}$ sections were prepared on a rotary microtome (RM2255, Leica, Germany), stained with cresyl violet, and scanned on an Axio Scan.Z1 (Zeiss, Germany) at 40x resolution.

\section{Brain volume}

We used preclinical 7T MRI (BioSpec 70/20 USR; Bruker, Ettlingen) with a $20 \mathrm{~cm}$ horizontal bore magnet and a $12 \mathrm{~cm}$ (inner diameter) shielded gradient with a $1 \mathrm{H}$-resonance-frequency of $300 \mathrm{MHz}$ and a maximum gradient strength of $440 \mathrm{mT} / \mathrm{m}$. Data acquisition was carried out with the Bruker software Paravision (version 6.0.1) at $35 \mu \mathrm{m}^{3}$ isovoxel resolution. The brain volume was visualized, outlined and measured in OsiriX Lite (version 8; osirix-viewer.com).

For comparing brain volume information to existing fish brain data, we obtained a digital copy of the FishBase ${ }^{25}$, kindly provided by Rainer Froese. To exclude potential records from larval or juvenile brains, we excluded entries that did not contain a literature reference or a body length measurement.

\section{Counting neurons in brain sections}

In order to estimate the total number of neurons, 28 brain slices ( $8 \mu \mathrm{m}$ thick, the 28 slices were $80 \mu \mathrm{m}$ apart from each other) were processed in the following manner: The images were convolved with a two-dimensional Gaussian envelope (full width at half-maximum: $1 \mu \mathrm{m}$ ). The images were individually thresholded, and the limits of the brain were manually determined on each of them. We finally counted the local maxima located within the brain regions above threshold, which yielded a total number of 89,327 cells. We then extrapolated to the entire 
brain volume. To prevent us from counting cells twice, we assessed the effective thickness of one brain slice to be its actual thickness $(8 \mu \mathrm{m})$ to which we added one cell diameter $(\sim 3 \mu \mathrm{m}$; after sample dehydration). We then multiplied the previous number by the total thickness of the brain $\left(280^{*} 8 \mu \mathrm{m}\right)$ divided by the effective thickness of all slices in which we counted cells $\left(28^{*} 11 \mu \mathrm{m}\right)$. We therefore estimate the DT brain to contain approximately 650,000 cells $\left(89,327^{*} 280 * 8 /\left(28^{*} 11\right)=649,651\right)$. In order to estimate the precision of the automatic cell detection scheme, we manually counted 4,486 cells in two slices. Within these slices we compared 10000 randomly chosen areas and computed the mean relative difference, given by $\left|N_{\text {auto }}-N_{\text {manual }}\right| / N_{\text {manual }}$, repeating the measurement when no cell was detected in the area. In order to correct for a putative effect of the size of the randomly picked region on the result, we repeated this operation with 10 different region sizes (from 11 to $111 \mu \mathrm{m}$, evenly spaced). Finally, we averaged over the resulting mean relative differences and obtained a precision estimation of $14 \%$.

\section{Shoaling and schooling}

Fish were tested in a behavioral room at $27^{\circ} \mathrm{C}$ (the same as in the housing tanks), in a transparent acrylic $24 \mathrm{~cm}$ square tank (experimental tank). The tank was filled with 1 I system water and was illuminated with infrared light from below. Behavioral videos were acquired with a CMOS camera at $25 \mathrm{fps}$, using an infrared longpass filter. On the days of the experiments, fish were removed from their housing tanks and placed in the experimental tank. Fish were left to habituate in the experimental tank for 10 minutes, after which a 10 minutes video recording was initiated. To test whether shoaling and schooling behavior in DT is visually mediated, we repeated the same test without room light. Each group of fish consisted of 12 male individuals.

Fish positions and orientations were extracted using custom written MATLAB (Mathworks) software. Background-subtracted frames were thresholded and binarized. Fish positions were computed as the coordinates of the centroids of each contiguous region. We also computed the weighted centroids as the centroids of the thresholded intensity-weighted image. The direction of each fish was computed from the vectors linking both centroids. Nearest-neighbor distances were calculated for each fish in each frame, and an average nearest-neighbor distance was calculated for a random subset of $m=6$ fish per frame (to ensure statistical 
independence, see ${ }^{51}$ ). The histogram of fish directions across all frames was computed after subtraction of the mean direction for each frame. For comparison, both nearest-neighbor distances and direction statistical analyses were run on a shuffled data set, where sets of $\mathrm{m}$ fish were randomly picked across frames in the entire video.

If fish were randomly distributed in the tank (complete spatial randomness or 'CSR' hypothesis), their nearest-neighbor distances (nnd) would be described by a Rayleigh distribution ${ }^{51}$. The mean and standard deviation of the averaged nnd values would be: $\mu=$ $\frac{1}{2 \sqrt{\lambda}}, \sigma=\sqrt{\frac{4-\pi}{4 m \pi \lambda}}$, where $\lambda$ is the fish density (number per area). Testing the CSR hypothesis against an experimentally measured average nearest-neighbor distance $d$ (Clark-Evans test),

we calculated the p-value as $p=\Phi\left(\frac{d-\mu}{\sigma}\right)$, where $\Phi$ is the cumulative distribution function of the normal distribution. Next, we tested if the distribution of measured nearest neighbor distances and the distances calculated from the shuffled data set were drawn from the same continuous distribution using the two-sided Kolmogorov-Smirnov test. Each shoaling and schooling observation (under light and dark condition) was replicated twice (always successfully).

\section{Code availability}

All custom code used for fish motion tracking, cell counting and vocalization analysis is available for download at https://github.com/danionella/schulze-henninger2018

\section{Recording and analysis of vocalizations}

DT vocalizations were recorded with a hydrophone (H2a-XLR, Aquarian Audio, WA, USA) and standard audio equipment (UR22 or UR44, Steinberg Media Technologies). To detect the discrete pulses of vocalizations, the waveform traces were bandpass-filtered $(1-10 \mathrm{kHz})$ and the waveform-envelope was extracted using a root-mean-square filter $(\tau=1.5 \mathrm{~ms})$. Single pulses were detected using the peak-detection algorithm of Todd ${ }^{52}$ and characteristics as time, peak amplitude and width were stored. Vocalizations are highly structured and pulses were generated predominantly at frequencies of about 60 and $120 \mathrm{~Hz}$. We defined a category „bursts" as groups of pulses that are separated by intervals shorter than $30 \mathrm{~ms}$ and „burst clusters" as groups of bursts that are separated by less than $300 \mathrm{~ms}$. We estimated the vocalization intensity (pulse peak amplitudes) from a recording of a community tank (26×22x16 
$\mathrm{cm}$ ) with 20 fish over two hours acquired with a data acquisition card (USB-6211, National Instruments, Austin, Texas, USA) connected to the UR44 amplifier for access to absolute voltage measurements.

To assess whether vocalizations are linked to aggression we performed behavioral analysis in a small tank $(24 \times 24 \mathrm{~cm})$ with shallow water. A group of four males was monitored over 24 hours using audio and video recordings. We analyzed three consecutive hours during a period of frequent vocalization (from $10 \mathrm{am}$ to $1 \mathrm{pm}$ ). We aimed to assess general aggression and therefore collected all aspects of fighting behavior (e.g., bites, chasing, striking and fleeing) in a single category. Fighting episodes were labeled manually using open source software (ANVIL, version 6, http://www.anvil-software.org/) using video only ("blindly", without listening to the audio track). Vocalizations were detected and quantified using software as described above and vocalization episodes were defined as the onset and offset of burst clusters. The event lists for fighting and vocalizations were transformed into binary vectors resampled at $100 \mathrm{~Hz}$ and the conditional probabilities $\mathrm{P}$ (vocalization|fighting) and $\mathrm{P}$ (fighting|vocalization) were calculated as $P(x \mid y)=x \cdot y / x \cdot x$, where $\cdot$ denotes the dot product. To assess the statistical significance of our measurements we generated a reference distribution of conditional probabilities from shuffled event lists $(n=20000)$ using the inter-event intervals and event durations of each list. Statistical significance was assessed using a one-sided onesample Student's t-test on the shuffled distribution and our measurement.

\section{Transgenesis}

The NeuroD:GCaMP6f plasmid was obtained from Andrew Prendergast and Claire Wyart, who kindly shared their plasmid prior to publication ${ }^{29}$. It is a bicistronic construct using mCherry under the crystallin promoter (expression in the lens) as an additional transgenesis marker. This plasmid was injected into 1-cell stage DT embryos in the following mixture: 35 $\mathrm{ng} / \mu \mathrm{L}$ Tol2 transposase mRNA, $15 \mathrm{ng} / \mu \mathrm{L}$ NeuroD:GCaMP6f, $0.1 \mathrm{M} \mathrm{KCl}, 0.1 \%$ phenol red. Larvae were screened for fluorescence and raised until adulthood ( $\geq 3$ months).

\section{Husbandry and Breeding}

DT were kept in commercial zebrafish aquaria (Tecniplast) with the following water parameters: $\mathrm{pH} 7.3$, conductivity $350 \mu \mathrm{S} / \mathrm{cm}$, temperature $27^{\circ} \mathrm{C}$. Larvae were co-cultured with L-type rotifers (Brachionus plicatilis, planktovie.biz) in static tanks from day 5 to $9{ }^{53}$. After 
entering water circulation on day 10, DT were fed with artemia as well as with dry food (Gemma Micro 75 until 4 weeks, then Gemma Micro 150; Skretting, USA). The fraction of larvae surviving into adulthood was $\sim 20 \%$. DT were bred in communal tanks of $20-40$ individuals. Because they are crevice spawners, they need narrow spaces for mating. We added $\sim 5 \mathrm{~cm}$ long silicone tubes to the breeding tanks, which were readily accepted as spawning environment (Supplementary Figure 1). Egg collection occurred during the first 3-5 hours of daylight. DT reach adulthood within 3 months. To establish transgenic lines, we combined up to $30 \mathrm{~F} 0$ animals in breeding tanks, collected all clutches and optically screened F1 individuals for germline transmission.

\section{Sequencing and library preparation}

DNA was obtained from 5 DT larvae at $5 \mathrm{dpf}$ using phenol-chloroform-isoamyl alcohol DNA extraction. The total number of reads in the obtained Illumina paired-end library was 1.347 billion (Macrogen, average insert size $500 \mathrm{bp}$ ). Raw reads were filtered by quality and trimmed of Illumina adaptors by fastq-mcf ${ }^{54}$ with parameters -C 1,000,000 -q 30 -u - I 35 -S. Filtered reads were mapped to the Danio rerio reference genome (GRCz10/danRer10) ${ }^{55}$ using bbmap ${ }^{56}$ with minratio parameter defining alignment tolerance to mismatches of 0.45 (range 0 to 1 ). Mapped reads were sorted and indexed with SAMtools ${ }^{57}$ and viewed in IGV ${ }^{58}$.

\section{Genome editing}

A consensus sequence of the first exon of the DT tyr gene was generated using previously obtained DT read mapping to the zebrafish reference. Sequences tyr gRNA1 (5'GGCTACTTCGGTGCCAACTG-3') and tyr gRNA2 (5'-TGAGATCCGGAAGCTGACAG-3') were chosen with the help of Harvard ChopChop online tool ${ }^{59}$ because of their predicted high efficiency.

We used the Alt-R CRISPR/Cas9 system and guide RNAs from IDT DNA. gRNA and tracrRNA were resuspended at $100 \mu \mathrm{M}$, mixed in equal amounts and aliquoted. gRNA:tracrRNA complexes were formed by heating the mixture to $95{ }^{\circ} \mathrm{C}$ for 2 min and cooling to room temperature. Afterwards, $10 \mu \mathrm{L}$ of the obtained complex mixture were combined with $1 \mu \mathrm{L}$ of the Cas9 buffer (200 mM HEPES-NaOH at pH 7.5, 1.5 M KCl) to make a guide RNA mixture. The injection mixture consisted of total $2 \mu \mathrm{L}$ guide RNA mixture, $1 \mu \mathrm{L}$ nuclease-free water and $1 \mu \mathrm{L}$ of $61 \mu \mathrm{M}$ Cas9 protein. 
To sequence the injected embryos, they were fixed with $100 \%$ methanol and digested for $1 \mathrm{~h}$ at $55^{\circ} \mathrm{C}$ with $10 \mu \mathrm{L}$ of $20 \mathrm{mg} / \mathrm{mL}$ Proteinase $\mathrm{K}$ stock solution (Roche) diluted 1:20 in $1 \mathrm{X} \mathrm{TE}$ solution. The proteinase $\mathrm{K}$ was then inactivated for $15 \mathrm{~min}$ at $95^{\circ} \mathrm{C}$. The digested embryos were shortly centrifuged and $1 \mu \mathrm{L}$ was used as a PCR template. The primers that were used for the amplification of the exon 1 of the tyr locus were TCGCTCTCTCCAGCAGTTCC and CTTCCAGGAGGAGAAGACTGAGG. The obtained PCR products were run on a $1 \%$ agarose gel, cut, purified, and cloned into a TOPO-TA vector (Invitrogen). 10 clones per band were sequenced and used for the alignment in Geneious (version 7.1.5).

Imaging

Animals were anesthetized in $120 \mathrm{mg} / \mathrm{l} \mathrm{MS-222.} \mathrm{Following} \mathrm{onset} \mathrm{of} \mathrm{anesthesia,} \mathrm{they} \mathrm{were}$ embedded in a drop of $3 \%$ agarose. As soon as the agarose gelled, hydrogel covering the mouth and gills was carefully removed with a scalpel without touching the fish. Once mouth and gills were free, animals were transferred to fish water containing a light sedation level of $30 \mathrm{mg} / \mathrm{l} \mathrm{MS}-222$. Two-photon images were acquired using a custom-built two-photon microscope controlled by Scanlmage 5.2.3 (Vidrio Technologies) and illuminated by a fspulsed $80 \mathrm{MHz}$ Ti:S laser (MaiTai DeepSee, SpectraPhysics). GCaMP6f fluorescence was imaged with a Nikon 16x 0.8 NA objective at an excitation wavelength of $930 \mathrm{~nm}$ and $\leq 10$ mW excitation power. Green fluorescence was collected using a 525/50 (Semrock) emission filter. 128x256 pixel frames were acquired at a rate of $13.4 \mathrm{~Hz}$. For Coumarin staining, $1 \mu \mathrm{g} / \mathrm{ml}$ Coumarin 6 (Sigma) was added to a housing tank overnight. Coumarin was excited at a wavelength of $820 \mathrm{~nm}$. To obtain the image in figure 4B, two fields-of-view were stitched using FIJI (http://fiji.sc). All animal experiments conformed to Berlin state, German federal as well as European Union animal welfare regulations and were approved by the LAGeSo, the Berlin admission authority for animal experiments.

Detailed information on experimental design and reagents used can be found in the Life Sciences Reporting Summary, which is published alongside this manuscript.

\section{Statistics}

In the shoaling and schooling experiment (Fig. 2A-C and Supplementary Figure 1) we tested the experimentally measured average nearest-neighbor distance $d$ (Clark-Evans test) against 
the complete-spatial-randomness hypothesis and calculated the p-value as $p=\Phi\left(\frac{d-\mu}{\sigma}\right)$, where $\Phi$ is the cumulative distribution function of the normal distribution. We also tested if the distribution of measured nearest neighbor distances and the distances calculated from the shuffled data set were drawn from the same continuous distribution using the two-sided Kolmogorov-Smirnov test. In the analysis of fighting behavior we assessed statistical significance of the measured co-occurrence of fighting and vocalizations against a shuffled distribution using a one-sided one-sample Student's t-test.

\section{Data Availability Statement}

Anatomical datasets are available at a GIN repository provided by the German Neuroinformatics Node ${ }^{22}$ at https://web.gin.g-node.org/judkewitzlab/schulze-henninger2018. Sequence alignment data have been deposited in the NCBI SRA database with the accession number SRP136594.

\section{Data Descriptors}

(1) High-resolution $35 \mu \mathrm{m}$ MRI of DT (83 MB zipped DICOM file): Data descriptor: Acquired with preclinical 7T MRT (BioSpec 70/20 USR; Bruker) with a $20 \mathrm{~cm}$ horizontal bore magnet and a $12 \mathrm{~cm}$ (inner diameter) shielded gradient with a $1 \mathrm{H}$-resonance-frequency of $300 \mathrm{MHz}$ and a maximum gradient strength of $440 \mathrm{mT} / \mathrm{m}$. Protocol: T1_FLASH_3D at $35 \mu \mathrm{m}^{3}$ isovoxel resolution. TR/TE $=50 / 9.5 \mathrm{~ms}$, flip angle $20^{\circ}, 8$ averages, FOV (field of view) coronal $14.0 \mathrm{x}$ 12.6 x $12.6 \mathrm{~mm}, \mathrm{MD}$ (matrix dimension) $400 \times 360 \times 360$, scan time 14h24min. Data can be explored with any freely available DICOM viewer. Filename: DT_MRI.zip

(2) Histological Nissl-stained sections of DT head (2.8 GB zip file): Data descriptor: 280 Nisslstained transverse sections ( $8 \mu \mathrm{m}$ thickness) of the DT head scanned on an Axio Scan.Z1 (Zeiss) at 40x resolution and stored in the CZI format of Zeiss, all metadata included. Data can be explored with any CZI viewer, such as 'ZEN' by Zeiss (tested on version 2.3 blue edition), FIJI and many others. A second file (TIFF) includes the aligned coronal sections of the brain. Data can be explored with any TIFF viewer, such as FIJI. Filename: DT_coronal_sections.zip

(3) DT sequencing reads aligned to the ZF genome (3.6 GB BAM file): deposited to the NCBI SRA database with accession number SRP136594. Data can be browsed with the freely 
available Integrative Genomics Viewer ${ }^{58}$ (see also Supplementary Figure 4).

Filename: DT_GRCz10_SRA.bam 


\section{Methods References}

51. Smith, T. E. Notebook on Spatial Data Analysis [online]. (seas.upenn.edu, 2016).

52. Todd, B. S. \& Andrews, D. C. The identification of peaks in physiological signals. Comput. Biomed. Res. 32, 322-335 (1999).

53. Martins, S. et al. Toward an Integrated Zebrafish Health Management Program Supporting Cancer and Neuroscience Research. Zebrafish 13, S-47-S-55 (2016).

54. Aronesty, E. Comparison of sequencing utility programs. The Open Bioinformatics Journal (2013).

55. Yates, A. et al. Ensembl 2016. Nucleic Acids Research 44, D710-D716 (2016).

56. Bushnell, B. BBMap short-read aligner, and other bioinformatics tools. (2015).

57. $\mathrm{Li}, \mathrm{H}$. et al. The Sequence Alignment/Map format and SAMtools. Bioinformatics 25, 2078-2079 (2009).

58. Robinson, J. T. et al. Integrative genomics viewer. Nat Biotech 29, 24-26 (2011).

59. Labun, K., Montague, T. G., Gagnon, J. A., Thyme, S. B. \& Valen, E. CHOPCHOP v2: a web tool for the next generation of CRISPR genome engineering. Nucleic Acids Research 44, W272-W276 (2016). 
Schulze et al. - Figure 1

A

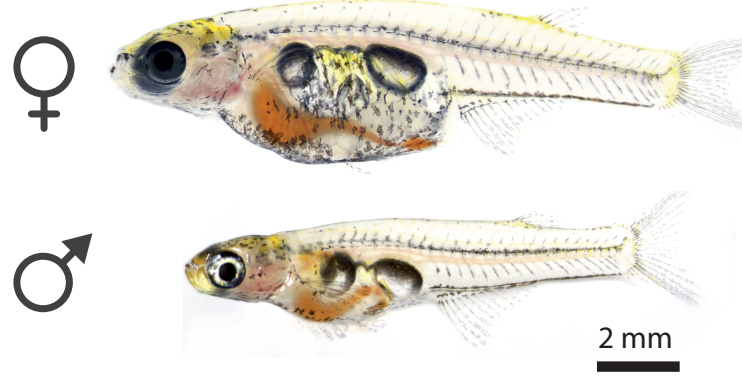

B

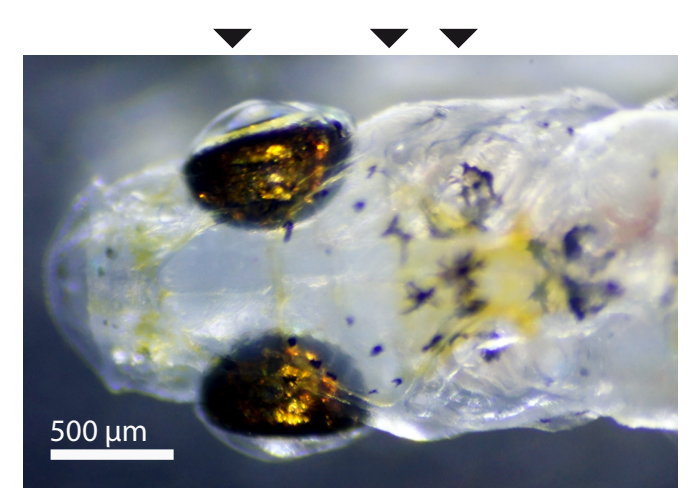

C

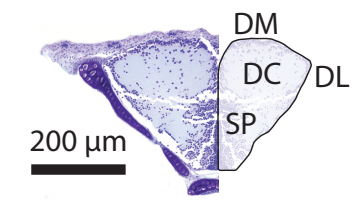

Forebrain

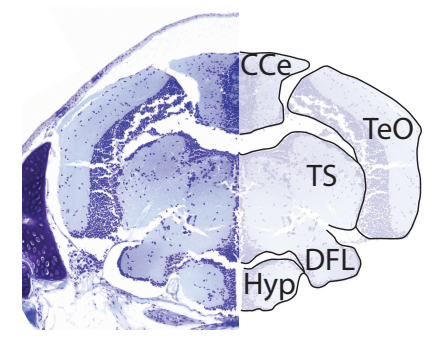

Midbrain

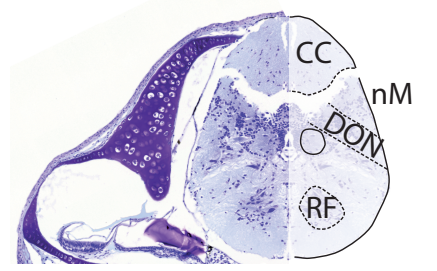

Hindbrain
D

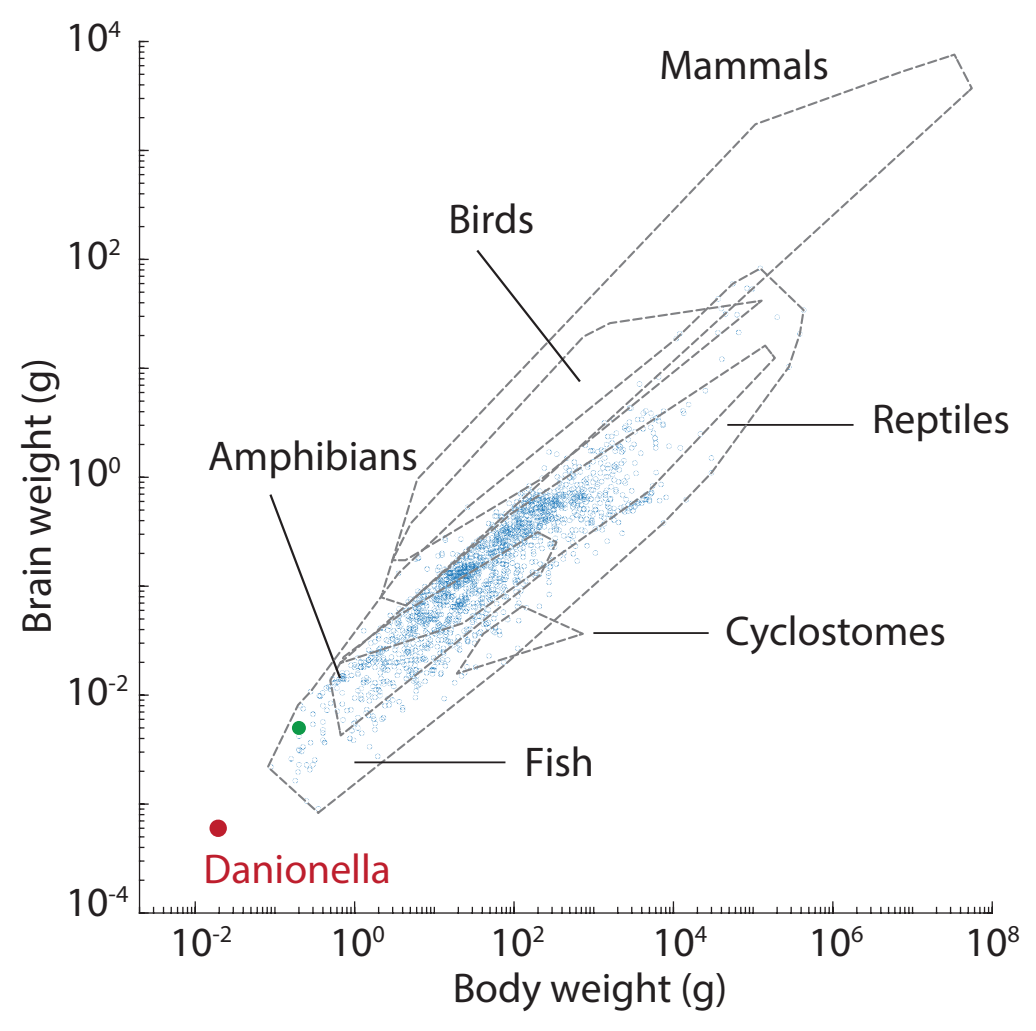


Schulze et al. - Figure 2

A

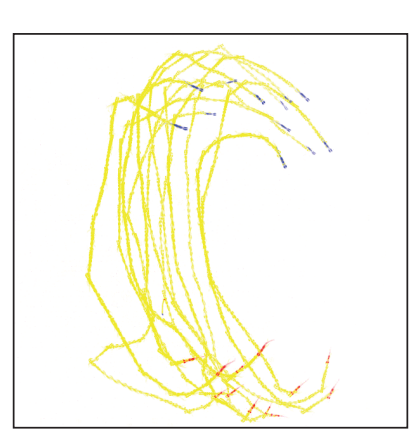

B

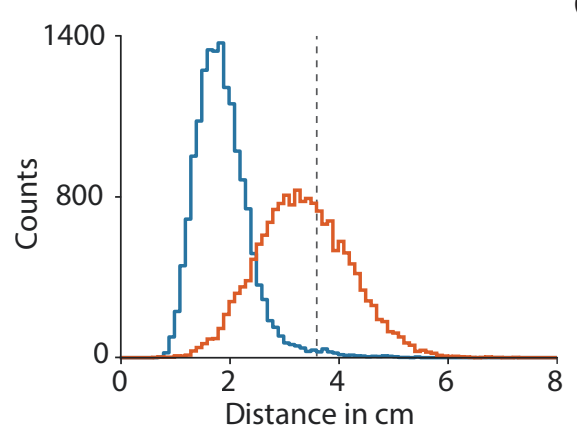

C

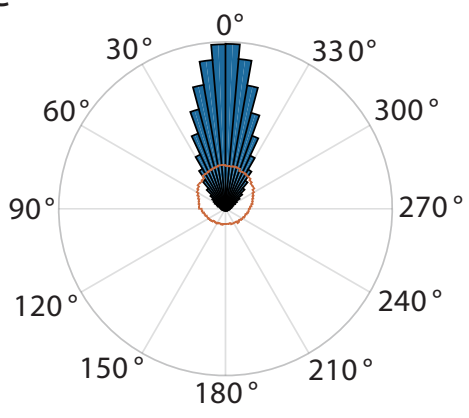

D

E
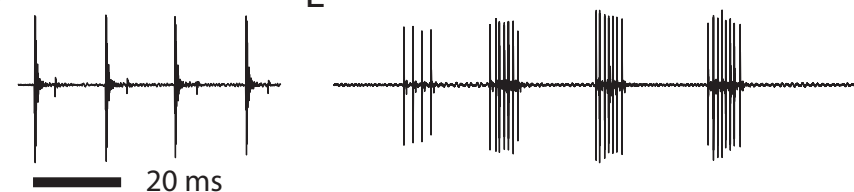

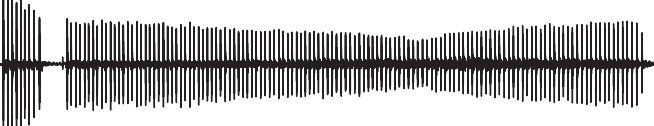

$100 \mathrm{~ms}$

F

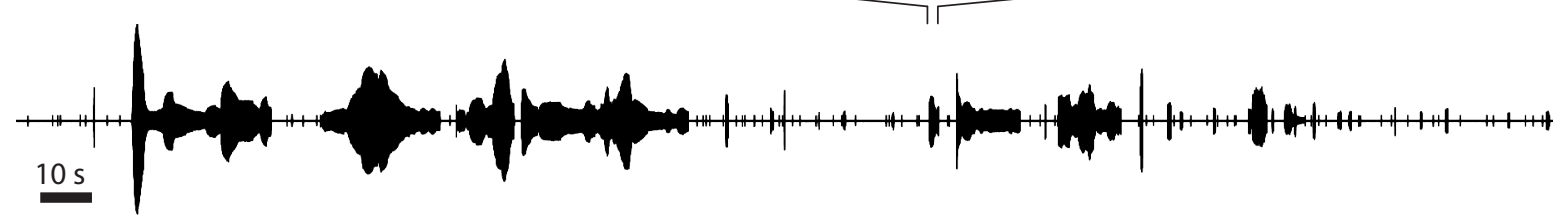

G
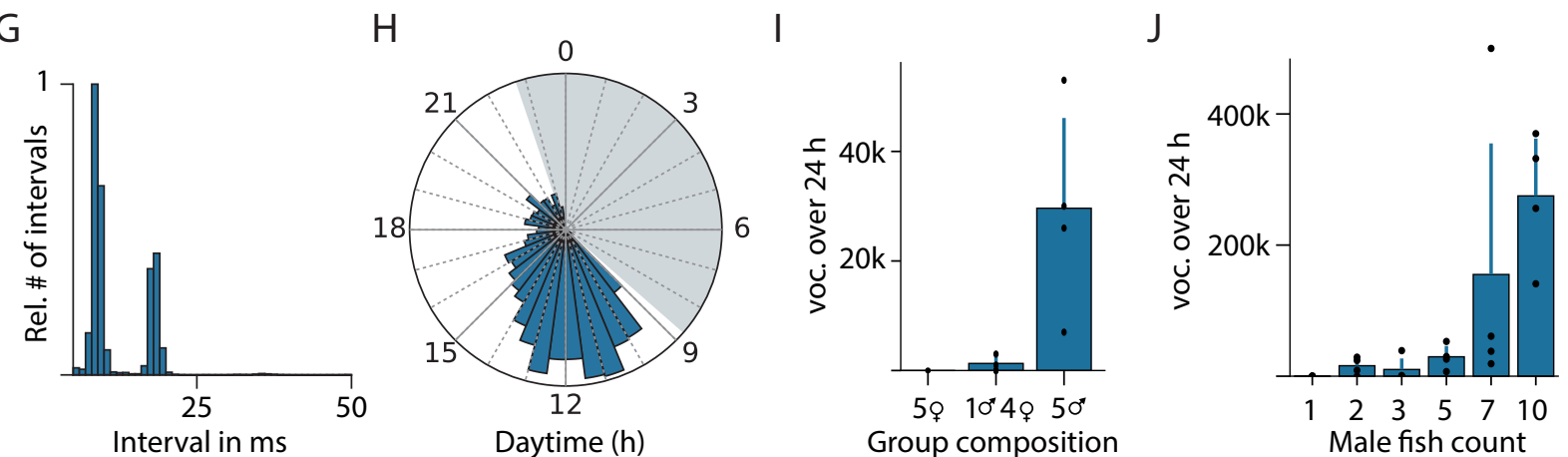
Schulze et al. - Figure 3

A

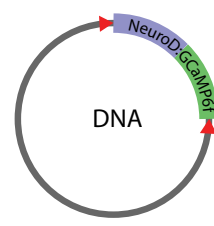

$+$

Tol2 mRNA

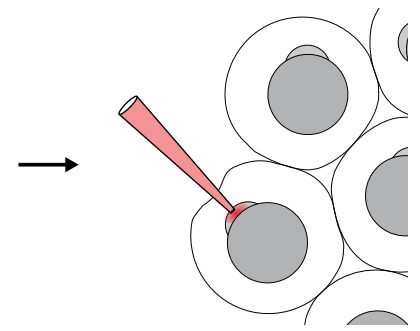

B Tol2 injected (FO)

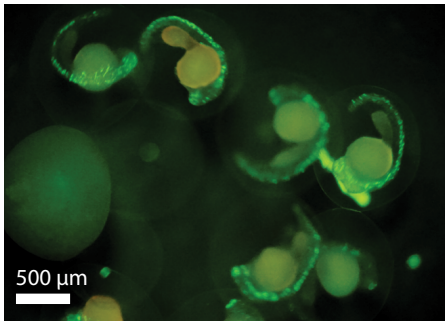

C F1

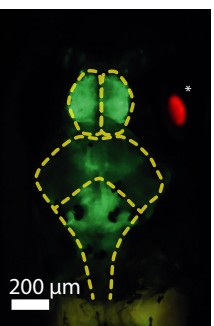

D

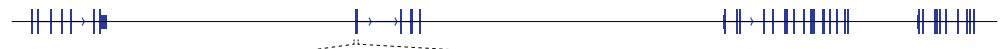

\begin{tabular}{lll}
$5^{\prime}$ UTR & Zebrafish tyr gene exon 1 & intron \\
\hline
\end{tabular}

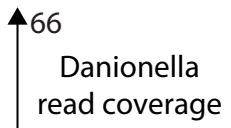


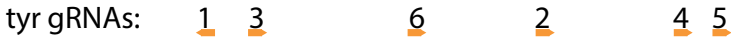

$\mathrm{F}$

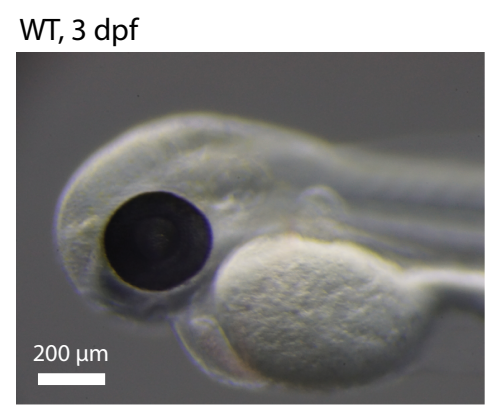

gRNA5+6 + CRISPR/Cas9, 3 dpf

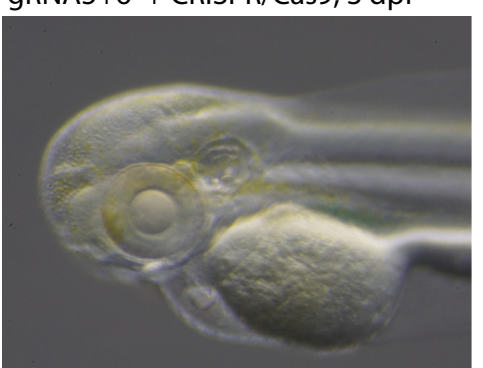

gRNA5+6 + CRISPR/Cas9, $3 \mathrm{dpf}$

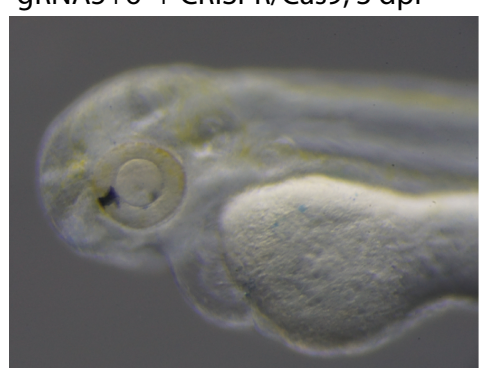

G

WT, $2 \mathrm{mpf}$

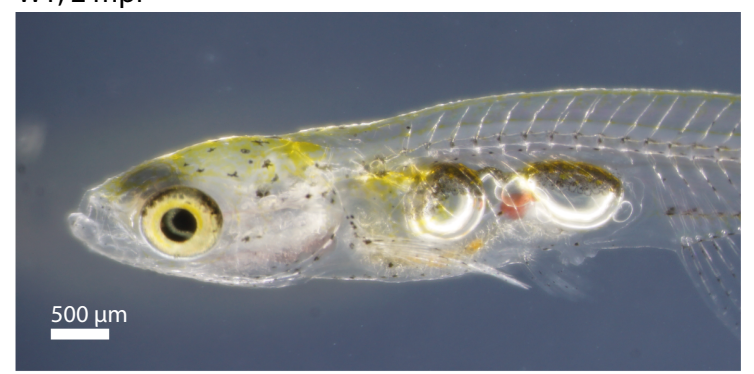

$\mathrm{E}$ Cas9+
gRNAs

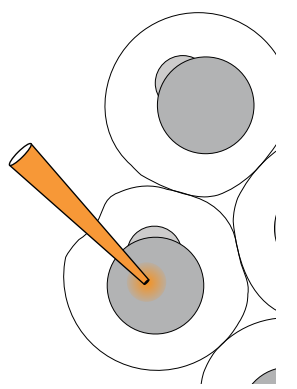

CRISPR tyr(-) F1, 2 mpf

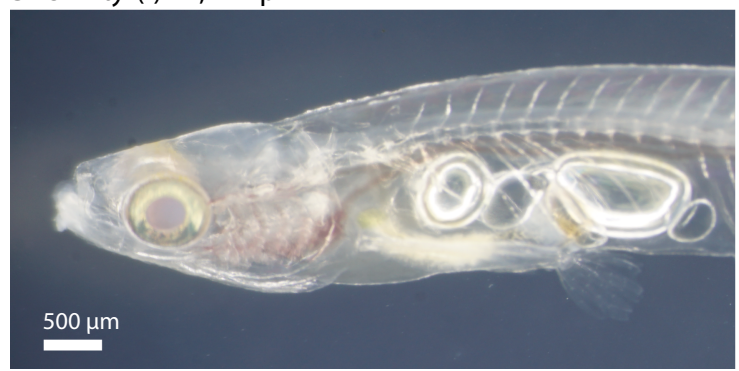


Schulze et al. - Figure 4
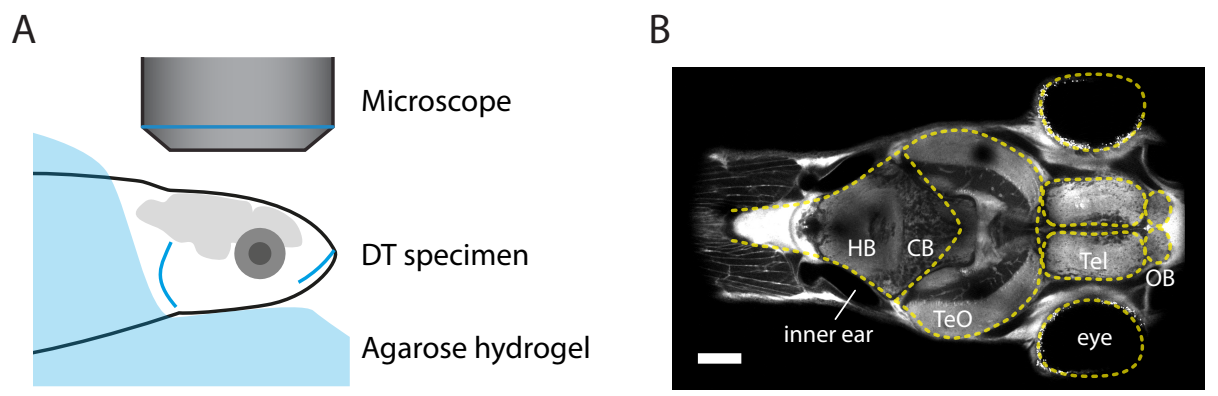

C

D

E
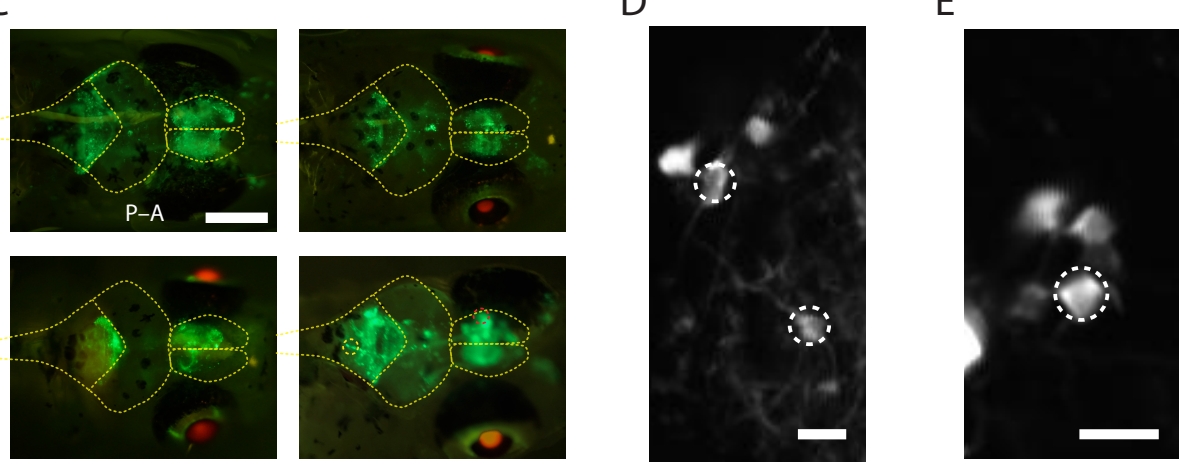

F

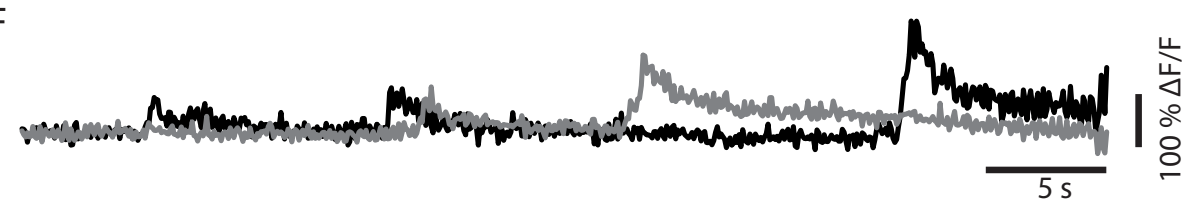

G

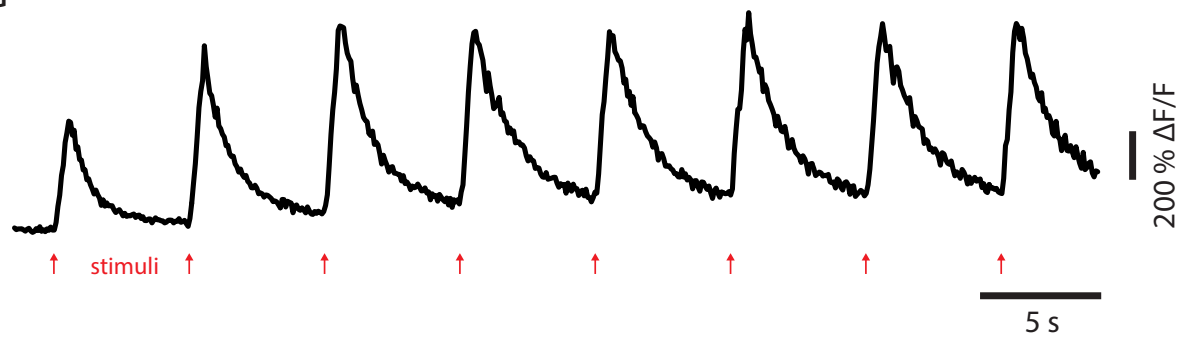

